\title{
Preparation of anti-HER-2 antibody PLGA polymer nano- ultrasound contrast agent In vitro targeting experiment
}

Ji Lin, Molly Stevens, and John Smith"

Department of Biochemistry, University of South Carolina, Columbia SC, 29208

\#Address: 631 Sumter Street, Columbia, SC 29208; Email: jsmith@medicine.usc.edu

\section{Abstract}

In this report, we demonstrated a novel method to prepare a hollow nano-targeted ultrasound contrast agent carrying anti-HER-2 antibody with high molecular weight polylactic acid-glycolic acid (PLGA) as a film-forming material, and to investigate in vitro targeting and imaging effects. We utilized the camphor as porogen, PLGA nanoultrasound contrast agent was prepared by modified double emulsion solvent evaporation method. The general characteristics were characterized by scanning electron microscope, transmission electron microscope and laser particle size analyzer. The angiography was performed by carbodiimide method. The anti-HER-2 antibody was used to prepare the PLGA-targeted nano-ultrasound contrast agent with anti-HER-2 antibody. The in-situ imaging ability was evaluated by laser confocal scanning microscopy. Results indicate that the average particle size of PLGA nano-ultrasound contrast agent was $(152.00 \pm 58.08) \mathrm{nm}$. The particles were regular spherical, uniform in size and good in dispersion. In vitro targeting experiments showed that PLGA-targeted contrast agents with anti-HER-2 antibodies were more strongly aggregated on the surface of breast cancer cells. In vitro imaging experiments showed that the PLGAtargeted nano-ultrasound contrast imaging showed a fine and uniform point-like hyperechoic echo, and no significant attenuation of the posterior echo. This study successfully produced a PLGA-targeted nano-ultrasound contrast agent with anti-HER2 antibody, which can specifically bind to breast cancer cells with high expression of HER-2 receptor in vitro, and the imaging effect in vitro is better.

\section{Introduction}

In recent years, high molecular weight polymers have been widely used in medical ultrasound contrast agents due to their biocompatibility, biodegradability and good filmforming properties [1], among which polylactic acid (PLA) and polylactic acid-hydroxyl groups Polylactic-co-glycolic acid (PLGA) is the most widely used. Microbubbles made of polymer as a film-forming material envelop air or perfluoropropane gas to produce strong echo scattering, which is an ideal ultrasonic contrast agent material. However, the tumor vascular endothelial space is $400-600 \mathrm{~nm}$. Traditional polymer microbubbles 
can only be confined to blood pool imaging, which limits their ability to detect extravascular tumor cells [2-3]. In this study, PLGA was used as the material, and camphor was used as the porogen. The nano-PLGA ultrasound contrast agent was prepared by the modified double emulsion solvent evaporation method, and the nanoparticles were combined with the fluorescently labeled anti-HER-2 antibody to prepare the targeted nano-ultrasound contrast agent. To detect its in vitro targeting ability and in vitro imaging effect on breast cancer SKBr3 cells.

\section{Materials and Method}

Main reagents: PLGA (50:50, Jinan Biotech Co., Ltd.); natural D (+)-camphor, polyvinyl alcohol PVA1788 low viscosity type (alcohol degree $87.0 \sim 89.0 \mathrm{~mol} / \mathrm{mol}$ ) and mannitol (Aladdin) Reagent Shanghai Co., Ltd.; Dichloromethane (Shanghai Runjie Chemical Reagent Co., Ltd.); Perfluoropropane Gas (Shanghai Renjieling Optical Instrument Co., Ltd.); Isopropyl Alcohol (Shenzhen Pharmaceutical Group Chemical Reagent Co., Ltd.); Ultrapure Water; Fluorescein isothiocyanate (FITC)-anti-HER-2 antibody (Shanghai Yixin Biotechnology Co., Ltd.); NHS/EDC (Billing reagent, Shanghai); phosphate buffered saline (PBS) Mercury Technology); paraformaldehyde, DAPI (Sigma, USA). Main instruments: ultrasonic cell pulverizer; RE-2000 rotary evaporator; Xiangyi $\mathrm{H}-1650$ high speed centrifuge; FD-1A-50 freeze dryer; field emission scanning electron microscope (S-4800, Japan); Malvern nanometer particle size Potential analyzer (ZEN3690, Germany); laser confocal microscope (Leica TCS SP5II, Germany); ion sputtering instrument (JFC-1100, Japan). Cell lines: breast cancer SKBr3 cells, MDAMB-231 cells, purchased from the Cell Resource Center of the Shanghai Institute of Biological Sciences, Chinese Academy of Sciences.

Preparation of PLGA nanoparticles by modified double emulsion solvent evaporation method: 1 accurately weigh $12.5 \mathrm{mg}$ of natural camphor, dissolve in $5 \mathrm{~mL}$ of dichloromethane; weigh PLGA 125 mg, also dissolve in dichloromethane; magnetically stir to fully dissolve, get Colorless transparent solution. 2 Take $1 \mathrm{~mL}$ of PVA solution $(3 \%, W / V)$, inject the above solution to obtain a water-oil two-phase solution; perform the first emulsification with an acoustic vibrometer under ice water bath conditions (130 W, on 4 s/off $2 \mathrm{~s}, 180 \mathrm{~s}$ ), obtaining a milky white primary emulsion. 3 The primary emulsion was injected into $20 \mathrm{~mL}$ of PVA solution $(3 \%$, W/V) for the second emulsification ( $130 \mathrm{~W}$, on $4 \mathrm{~s} /$ off $2 \mathrm{~s}, 180 \mathrm{~s}$ ). 4 Pour the secondary emulsion into 100 $\mathrm{mL}$ of isopropanol solution $(5 \%, \mathrm{~V} / \mathrm{V})$ and spin to evaporate to evaporate the solvent and solidify the surface of the particles. 5 The mixture obtained by rotary evaporation was placed in a $15 \mathrm{~mL}$ centrifuge tube and centrifuged at $12000 \mathrm{r} / \mathrm{min}$ for $10 \mathrm{~min}$; the supernatant was discarded, and the white precipitate was re-dissolved in ultrapure water and centrifuged again, 3 times; A small amount of mannitol was added to the precipitate, dispersed in $1 \mathrm{~mL}$ of ultrapure water, and wrapped in aluminum foil and 
placed in a refrigerator at $-20{ }^{\circ} \mathrm{C}$ for freezing. 6 It was placed in a freeze dryer for $24 \mathrm{~h}$ to freeze-dry, to obtain a white powder, and a vacuum was applied to inject perfluoropropane gas. 7 Keep the product sealed and protected from light in a refrigerator at $4{ }^{\circ} \mathrm{C}$.

The morphology of PLGA hollow nanoparticles was observed by field emission scanning electron microscopy. The PLGA hollow nanoparticles were dispersed in ultrapure water. Appropriately dispersed samples were applied to the rough surface of aluminum foil to prepare dried samples and then sprayed with an ion sputter. Gold, under the scanning electron microscope, observed the particle size, morphology, surface and dispersibility. The PLGA hollow nanoparticles were dispersed in ultrapure water by a nano-particle size potentiometer, and the hydrated particle size and polydispersity index of PLGA hollow nanoparticles were measured at room temperature. It was confirmed by transmission electron microscopy whether the particles had a hollow structure: the PLGA particles were dispersed and dropped onto a copper film covered with a carbon film, and dyed with a freshly prepared phosphotungstic acid solution $(1 \%, \mathrm{~W} / \mathrm{V})$ under a transmission electron microscope. Observe.

The PLGA nanoparticles $(1 \mathrm{mg} / \mathrm{mL})$ dispersion was incubated with the coupling activator NHS/EDC for $30 \mathrm{~min}$ at room temperature, centrifuged at $16000 \mathrm{r} / \mathrm{min}$ for 10 min; the supernatant was discarded and reconstituted in PBS for 2 times. The unreacted coupling activator NHS/EDC is removed. Disperse the activated PLGA nanoparticles in $200 \mu \mathrm{L}$ PBS, add $25 \mu \mathrm{L}$ of $1 \mu \mathrm{g} / \mu \mathrm{L}$ FITC-anti-HER-2 antibody, mix well, incubate for $30 \mathrm{~min}$, centrifuge at $16000 \mathrm{r} / \mathrm{min}$ for $10 \mathrm{~min}$; disperse the precipitate In PBS, repeat 2 times, remove free antibody, obtain targeted nano-ultrasound contrast agent, and disperse in $50 \mu \mathrm{L}$ PBS for use.

Preparation of cell culture and slides Two breast cancer cells (SKBr3 cells, MDA-MB231 cells) were cultured at $37^{\circ} \mathrm{C}, 5 \% \mathrm{CO}$, and fully saturated humidity, and cultured in DMEM containing $10 \%$ fetal bovine serum. For SKBr3 cells, MDA-MB-231 cells were cultured in L-15 medium containing 10\% fetal bovine serum. Both cells were cultured at a density of $1 \times 104 /$ dish in a Petri dish with a thickness of $0.17 \mathrm{~mm}$, and the experiment was started 24 hours after the cells were attached.

Take SKBr3 cells and MDA-MB-231 cells one by one, add $25 \mu \mathrm{L}$ FITC-anti-HER-2 antibody separately; take SKBr3 fine-carrying anti-HER-2 antibody PLGA polymer nanoultrasound contrast agent preparation and its in vitro targeting Experiments - $108 \cdot$ In June 2014, Volume 23, Phase 2, and MDA-MB-231 cells were each supplemented with $25 \mu \mathrm{L}$ of targeted nano-ultrasound contrast agent. All 4 cells were incubated for 20-30 min, then washed 3 times with PBS to remove unbound antibody and targeted nano- 
ultrasound contrast agent. Fixation was carried out with a $4 \%$ paraformaldehyde solution and stained with DAPI. The above operations are all carried out in the dark.

In vitro imaging effect of targeting PLGA nanoparticles: accurately weigh a certain amount of PLGA hollow nanoparticle powder, prepare a dispersion of $1 \mathrm{mg} / \mathrm{mL}$, take 5 $\mathrm{mL}$ of transparent plastic sample tube filled with rubber stopper, and take $5 \mathrm{~mL}$ at the same time Airless water was injected into the same sample tube as a blank control group. With airless water as the sound-transparent window, real-time gray-scale imaging (central frequency $22 \mathrm{MHz}$ UHF probe with a mechanical index of 0.06 ) was performed with a Yum MyLab Twice ultrasound system.

\section{Results and Discussion}

The prepared PLGA nanoparticle dispersion sample was dried and sprayed with gold and observed under a scanning electron microscope. The particles were spherical, the surface was smooth, the shape was regular, the dispersion was good, and no agglomeration was observed between the particles (Fig. 1). The average particle size of the particles measured by the nanoparticle size potential analyzer was $(152.00 \pm 58.08)$ $\mathrm{nm}$, and the polydisperse index (PDI) was 0.221 , indicating that the particle size distribution was uniform (Fig. 2, 3).

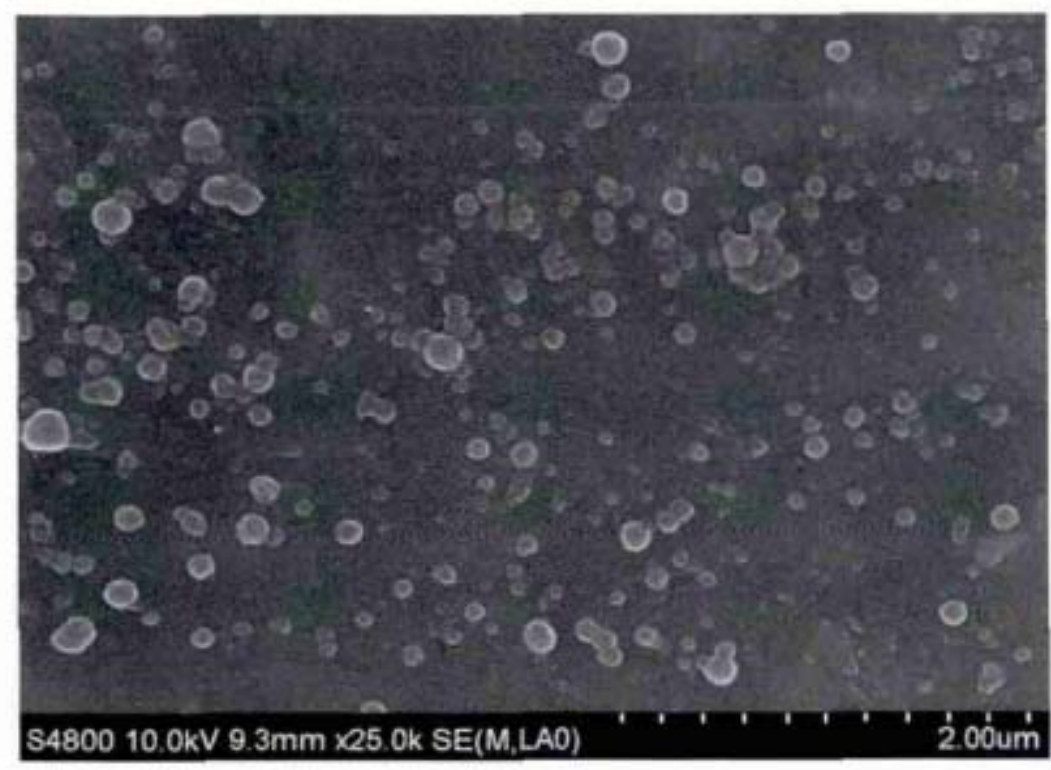

Figure 1. SEM Images of nanoparticles

As shown in Fig. 4A, the success rate of FITC-anti-HER-2 antibody and SKBr3 cells was observed under laser confocal microscopy. The nucleus was stimulated by DAPI staining to show blue fluorescence, and the cell membrane was excited to obtain a ring or a point. Green fluorescence, indicating that the antibody successfully binds to the cell 
membrane. Figure 4B shows that some of the SKBr3 cells bind to the target nanoultrasound contrast agent, and the cell membrane shows a dot-like green fluorescence, indicating that the targeted nano-ultrasound contrast agent is more and firmly attached to the surface of breast cancer SKBr3 cells and is not eluted by PBS. In Fig. 4C and Fig. 4D, no adhesion of antibody or targeted polymer contrast agent was observed on the surface of MDA-MB-231 cell membrane, which further confirmed that the targeted contrast agent has higher expression of HER-2 receptor-expressing breast cancer cells in vitro. Strong specific affinity.

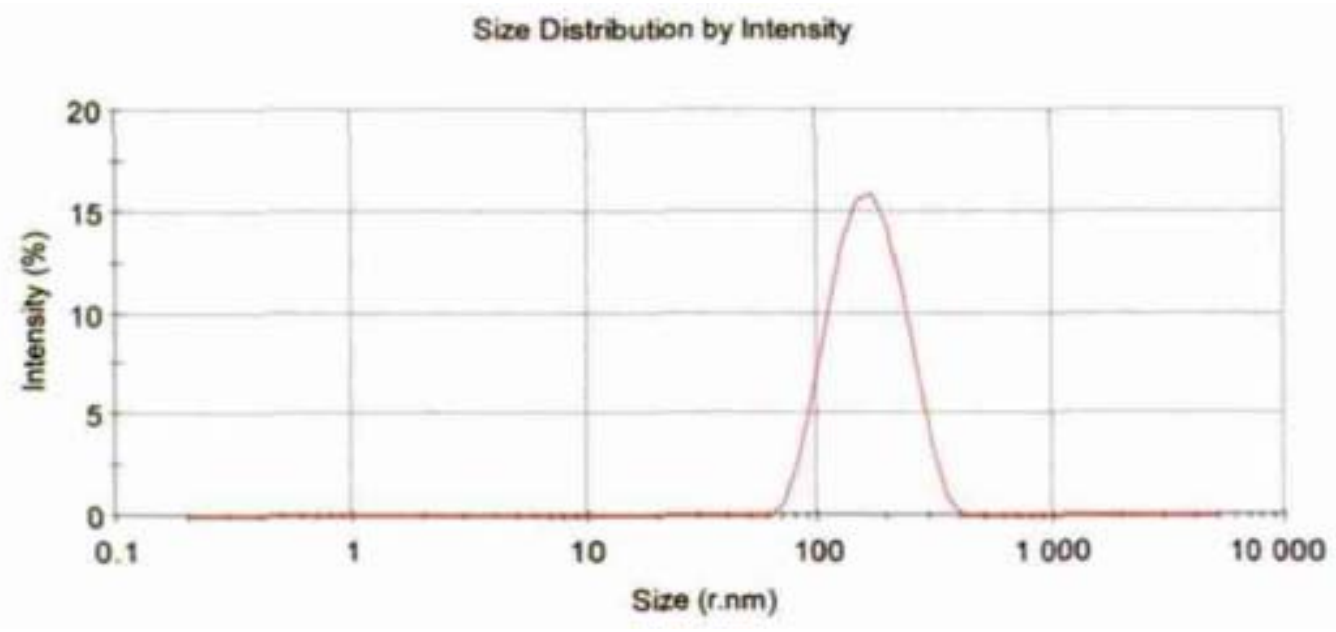

Figure 2. Nanoparticle size analysis by dynamic light scattering

The nano-targeted contrast agent with a concentration of $1 \mathrm{mg} / \mathrm{mL}$ was filled into a sample tube with a volume of $5 \mathrm{~mL}$, and the sample tube containing $5 \mathrm{~mL}$ of degassed water was used as a reference, and the targeted nano-polymer ultrasound was observed with a high-frequency linear array probe. The ability of the contrast agent to develop in vitro. As a result, as shown in Fig. 5, the PLGA contrast agent group showed a point-like hyperechoic, which was fine and uniform, and the water of the control group showed an anechoic fluid-free area. 


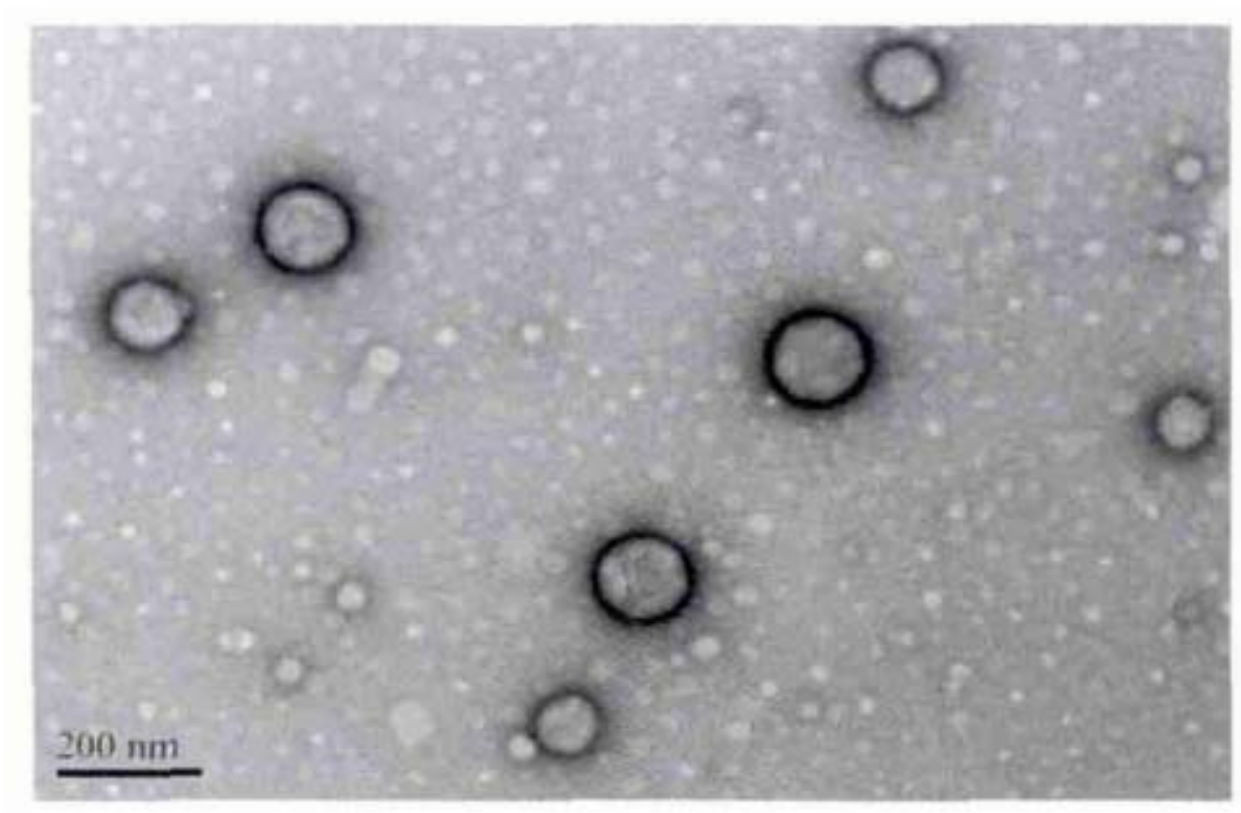

Figure 3. TEM images of nanoparticles loaded with fluorophore

The ultrasonic contrast agent prepared by using the high-molecular polymer PLGA as a film-forming material is biocompatible and biodegradable, and can be metabolized into non-toxic carbon dioxide and water in the living body, and is harmless to the living body, and is ideal. Ultrasound contrast material has a good application prospect [4-5]. 

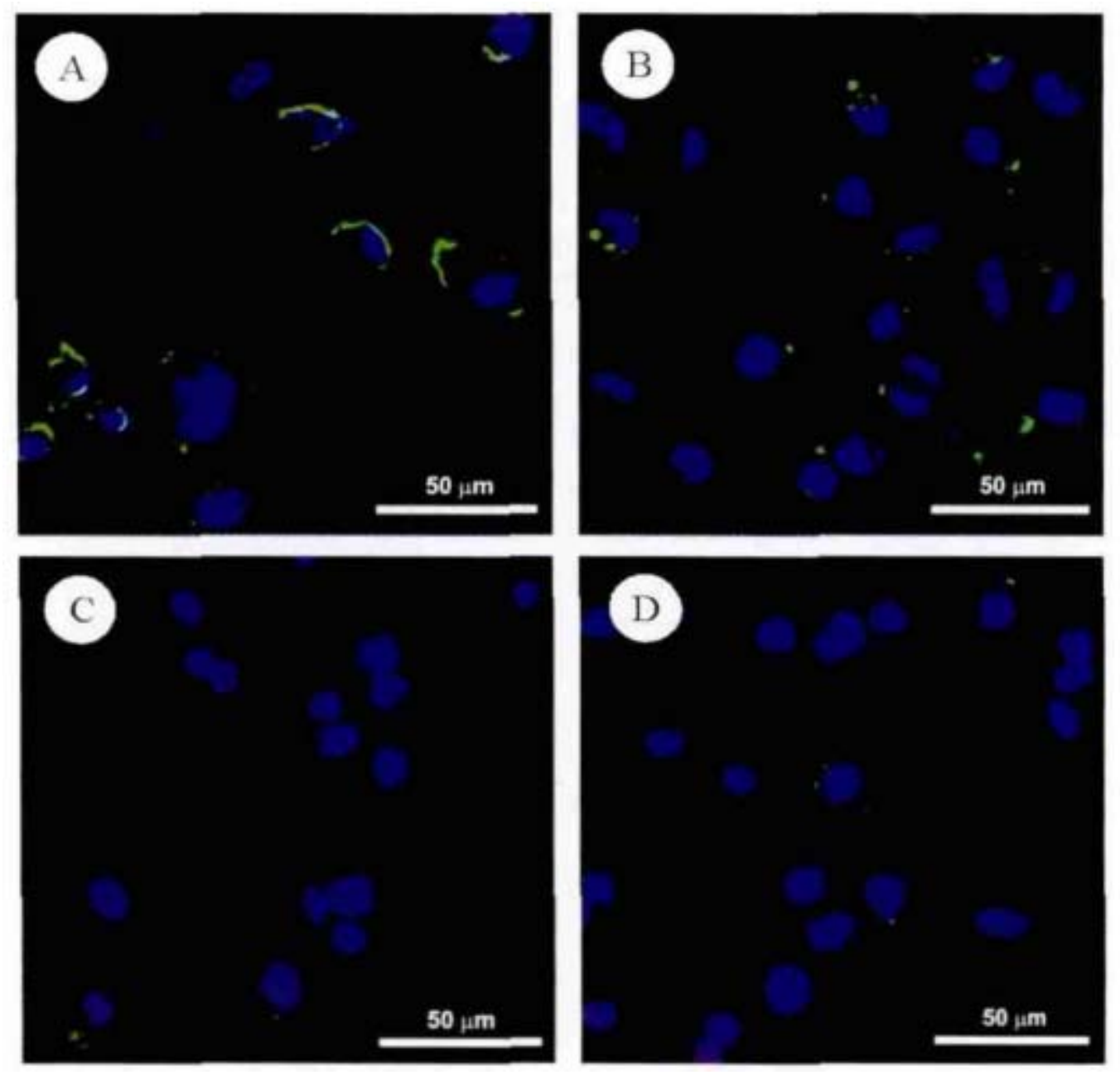

Figure 4. Cell uptake measured by fluorescent microscopy

In this study, the camphor was dissolved in the primary emulsifier, and the PLGA particles coated with camphor were prepared by the modified double emulsion solvent evaporation method. After freeze-drying, the camphor sublimed and filled with perfluoropropane gas to obtain PLGA nano-ultrasound contrast agent. The surface of the particle is smooth and regular, the average particle size is $(152.00 \pm 58.08) \mathrm{nm}$, the particle size is monomodal, and the PDI is 0.221 . It can theoretically penetrate the tumor vascular endothelium $(400-600 \mathrm{~nm})$ into the tumor tissue. It is possible to visualize extravascular target tissue through "passive targeting", thereby overcoming the limitations of traditional microbubble contrast agents in intravascular imaging [6]. The surface of the nano-contrast prepared by PLGA contains a large amount of carboxyl groups, and the active substances such as monoclonal antibodies, ligands, and various specific short peptides are mostly proteins, which themselves contain a large amount of amino groups, and carboxyl groups and amino groups can be coupled by covalent bonds. In this study, EDC/NHS was used as a coupling activator to bind the carboxyl group on the activated PLGA to the amino group on the monoclonal antibody, and the targeted PLGA nano-sonography with anti-HER-2 antibody was successfully 
prepared by the carbodiimide method. And demonstrated its ability to specifically bind to breast cancer SKBr3 cells with high expression of HER-2 receptor. The nano-ultrasound contrast agent carrying the anti-HER-2 antibody is expected to be actively targeted in the body, so that the ultrasound contrast agent can be concentrated in the breast cancer tissue at a higher concentration to achieve the purpose of enhanced orientation.
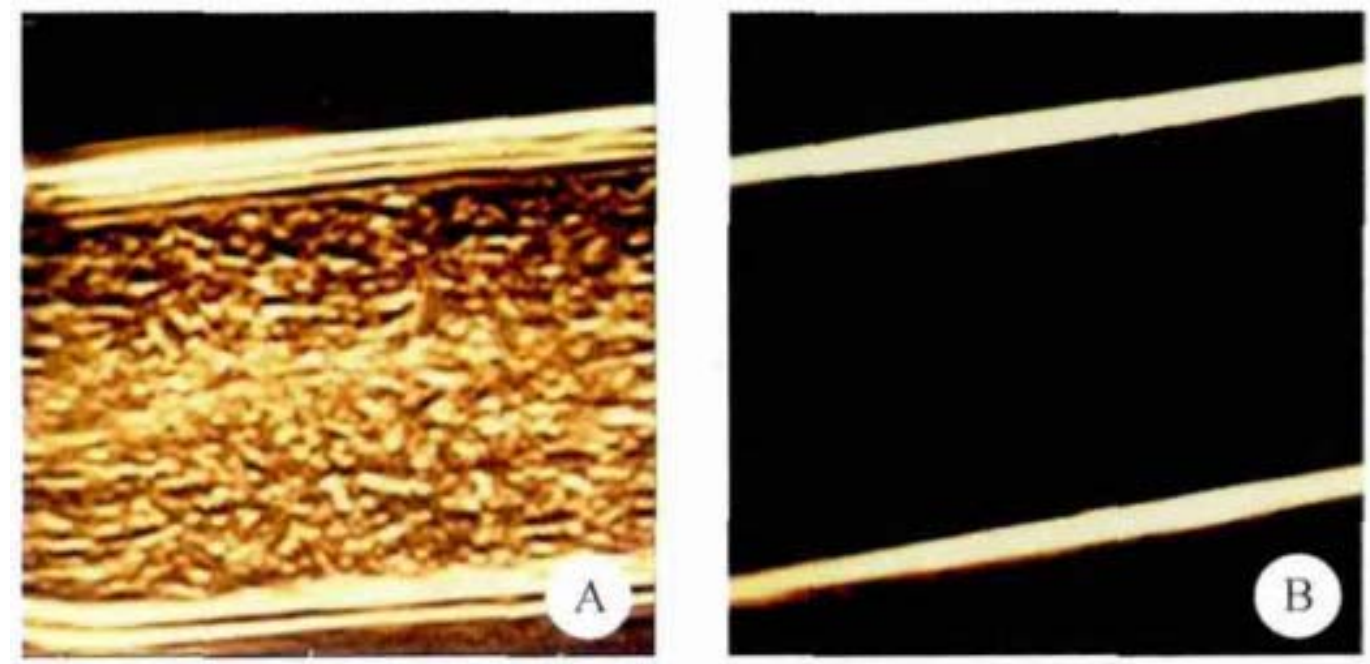

Figure 5. In-situ imaging of PLGA nanoparticles.

Transmission electron microscopy confirmed that the nano-particles prepared in this study have a cavity structure, the presence of a cavity structure and the introduction of perfluoropropane gas can produce strong echo scattering. PLGA nano-ultrasonic agents differ greatly from conventional microbubble contrast agents in their imaging patterns, which are not suitable for imaging of traditional microbubble contrast agents on ultrasound systems. Preliminary in vitro ultrasound imaging experiments have shown that PLGA-targeted nano-contrast agents have better imaging effects under highfrequency ultrasound conditions ${ }^{1-14}$.

The carboxylic acid bond $(-\mathrm{COOH})$ on the surface of PLGA nanoparticles and the internal cavity make it not only a targeted ultrasound contrast agent, but also a drug on the surface of the particle or a quantitative drug encapsulated inside the particle to achieve targeted tumor diagnosis and The purpose of treatment [7] lays a good foundation for the further development of PLGA nano-targeted drug-loaded contrast agents with dual functions of diagnosis and treatment.

\section{Conclusion}

In summary, this study successfully prepared a PLGA-targeted nano-ultrasound contrast agent with anti-HER-2 antibody, which can specifically bind to breast cancer cells with high expression of HER-2 receptor in vitro, and in vitro imaging effect. better. Future efforts will focus on translating this research to the clinic. 


\section{References}

1. Laird, P. W.; Zijderveld, A.; Linders, K.; Rudnicki, M. A.; Jaenisch, R.; Berns, A., Simplified mammalian DNA isolation procedure. Nucleic acids research 1991, 19 (15), 4293.

2. Boussif, O.; Lezoualc'h, F.; Zanta, M. A.; Mergny, M. D.; Scherman, D.; Demeneix, B.; Behr, J.-P., A versatile vector for gene and oligonucleotide transfer into cells in culture and in vivo: polyethylenimine. Proceedings of the National Academy of Sciences 1995, 92 (16), 7297-7301.

3. Kenworthy, A. K.; Hristova, K.; Needham, D.; Mclntosh, T. J., Range and magnitude of the steric pressure between bilayers containing phospholipids with covalently attached poly (ethylene glycol). Biophysical journal 1995, 68 (5), 1921-1936.

4. Krieg, A. M.; Yi, A.-K.; Matson, S.; Waldschmidt, T. J.; Bishop, G. A.; Teasdale, R.; Koretzky, G. A.; Klinman, D. M., CpG motifs in bacterial DNA trigger direct B-cell activation. Nature 1995, 374 (6522), 546.

5. Yu, Y.-C.; Roontga, V.; Daragan, V. A.; Mayo, K. H.; Tirrell, M.; Fields, G. B., Structure and dynamics of peptide- amphiphiles incorporating triple-helical proteinlike molecular architecture. Biochemistry 1999, 38 (5), 1659-1668.

6. Zhang, R.; Billingsley, M. M.; Mitchell, M. J., Biomaterials for vaccine-based cancer immunotherapy. Journal of Controlled Release 2018.

7. Zhang, R.; Kramer, J. S.; Smith, J. D.; Allen, B. N.; Leeper, C. N.; Li, X.; Morton, L. D.; Gallazzi, F.; Ulery, B. D., Vaccine Adjuvant Incorporation Strategy Dictates Peptide Amphiphile Micelle Immunostimulatory Capacity. The AAPS journal 2018, 20 (4), 73.

8. Zhang, R.; Leeper, C. N.; Wang, X.; White, T. A.; Ulery, B. D., Immunomodulatory vasoactive intestinal peptide amphiphile micelles. Biomaterials science 2018.

9. Zhang, R.; Leeper, C. N.; Wang, X.; White, T. A.; Ulery, B. D., Immunomodulatory vasoactive intestinal peptide amphiphile micelles. Biomaterials science 2018, 6 (7), 1717-1722.

10. Zhang, R.; Morton, L. D.; Smith, J. D.; Gallazzi, F.; White, T. A.; Ulery, B. D., Instructive Design of Triblock Peptide Amphiphiles for Structurally Complex Micelle Fabrication. ACS Biomaterials Science \& Engineering 2018.

11. Zhang, R.; Smith, J. D.; Allen, B. N.; Kramer, J. S.; Schauflinger, M.; Ulery, B. D., Peptide Amphiphile Micelle Vaccine Size and Charge Influence the Host Antibody Response. ACS Biomaterials Science \& Engineering 2018.

12. Zhang, R.; Smith, J. D.; Allen, B. N.; Kramer, J. S.; Schauflinger, M.; Ulery, B. D., Peptide Amphiphile Micelle Vaccine Size and Charge Influence the Host Antibody Response. ACS Biomaterials Science \& Engineering 2018, 4 (7), 2463-2472.

13. Zhang, R.; Ulery, B. D., Synthetic vaccine characterization and design. Journal of Bionanoscience 2018, 12 (1), 1-11.

14. Smith, J. D.; Cardwell, L. N.; Porciani, D.; Nguyen, J. A.; Zhang, R.; Gallazzi, F.; Tata, R. R.; Burke, D. H.; Daniels, M. A.; Ulery, B. D., Aptamer-displaying peptide amphiphile micelles as a cell-targeted delivery vehicle of peptide cargoes. Physical biology 2018, 15 (6), 065006. 